Cahiers de recherches médiévales

\title{
La bibliothèque de Saint-Victor et les Lumières
}

\section{Geneviève Artigas-Menant}

\section{(2) OpenEdition}

Journals

Édition électronique

URL : https://journals.openedition.org/crm/11529

DOI : $10.4000 / \mathrm{crm} .11529$

ISSN : 1955-2424

Éditeur

Honoré Champion

Édition imprimée

Date de publication : 15 juin 2009

Pagination : 271-281

ISSN : 1272-9752

\section{Référence électronique}

Geneviève Artigas-Menant, "La bibliothèque de Saint-Victor et les Lumières », Cahiers de recherches médiévales [En ligne], 17 | 2009, mis en ligne le 15 juin 2012, consulté le 15 décembre 2022. URL : http://journals.openedition.org/crm/11529; DOI : https://doi.org/10.4000/crm.11529 


\title{
RM
}

\section{La bibliothèque de Saint-Victor et les Lumières}

\begin{abstract}
The accounts of many $17^{\text {th }}$-and $18^{\text {th }}$-century writers (Giovanni Paolo Marana, Robert Challe, Montesquieu) and of foreign travellers (the Englishman Martin Lister, the Italian Alfonso Bonfioli Malvezzi) indicate that Saint-Victor was among the most famous and most frequented Parisian libraries. Modern scholars had already located five "clandestine philosophical manuscripts" belonging to Saint-Victor. This was the name given to unpublished, anti-Christian texts - sometimes deist, sometimes atheistic and materialistic, always violently subversive - from which the philosophers of the Enlightenment drew inspiration. This article discloses the existence of five additional manuscripts of the same kind in the catalogues of Saint-Victor maintained at the Bibliothèque Mazarine. Their titles demonstrate that the canons of Saint-Victor were capable of making relevant selections among the manifestos of the most daring contemporary thought and were thus far from retreating to past knowledge.
\end{abstract}

Résumé: Les témoignages d'écrivains des XVII et XVIII siècles (Giovanni Paolo Marana, Robert Challe, Montesquieu) et de voyageurs étrangers (l'anglais Martin Lister, l'italien Alfonso Bonfioli Malvezzi ) montrent que Saint-Victor était une des bibliothèques parisiennes les plus célèbres et les plus visitées. Les chercheurs modernes avaient déjà repéré cinq «manuscrits philosophiques clandestins» ayant appartenu à Saint-Victor. On appelle ainsi des textes antichrétiens inédits, tantôt déistes, tantôt athées et matérialistes, toujours violemment subversifs, dont se sont inspirés les philosophes des Lumières. Cet article révèle l'existence, dans les catalogues de Saint Victor conservés à la Mazarine, de cinq autres manuscrits du même corpus. Leurs titres prouvent que les chanoines de Saint-Victor avaient su opérer un choix pertinent dans les manifestes de la pensée contemporaine la plus hardie et étaient donc éloignés d'un repli sur les savoirs du passé.

La bibliothèque de Saint-Victor est-elle, au XVIII ${ }^{\mathrm{e}}$ siècle, «le symbole d'un monde de clercs ayant renoncé à tout dynamisme intellectuel et vivant replié sur un savoir suranné »? Pour répondre à cette question posée par Isabelle Guyot-Bachy, je tenterai d'étudier les traces de l'attention portée par les chanoines à la critique antireligieuse contemporaine sous sa forme la plus radicale et la plus caractéristique de la naissance des Lumières : les manuscrits philosophiques clandestins.

Ce que l'on appelle manuscrits philosophiques clandestins est un ensemble épars de textes antireligieux rédigés à la fin du $\mathrm{XVII}^{\mathrm{e}}$ siècle et dans la première moitié du XVIII ${ }^{\mathrm{e}}$ siècle. Leur existence a été découverte en 1912 par Gustave Lanson qui, dans un célèbre article fondateur de la Revue d'Histoire littéraire de la France $^{1}$, faisait des hypothèses et lançait un programme de recherche dans les fonds manuscrits des bibliothèques. Il dénombrait une centaine de copies manuscrites représentant trente-cinq textes différents, à Paris et en province. D'après lui, si la

${ }^{1}$ G. Lanson, «Questions diverses sur l'histoire de l'esprit philosophique en France avant $1750 », R H L F, 1912$, p. 1-29, 293-317.

Cahiers de Recherches Médiévales, 17, 2009 
circulation et la diffusion de ces manuscrits était confirmée, on aurait la preuve que «le développement de l'incrédulité radicale et violente était antérieur à 1750 » et que c'était dans la «masse anonyme» des manuscrits qu'il faudrait «aller chercher la pensée dangereuse et neuve d'où se sont élevés les grands écrivains ». En 1938 Ira $\mathrm{O}$. Wade ${ }^{2}$ confirmait l'hypothèse de Lanson avec cent deux titres au lieu de trentecinq et quatre cent trois copies au lieu de cent dix en France et à l'étranger. En 1996 le bilan de Miguel Benítez ${ }^{3}$ faisait passer ces deux chiffres respectivement à cent quatre-vingt-douze titres et près de deux mille copies. Depuis 1992, La Lettre clandestine $^{4}$ dresse annuellement de nouvelles listes.

Cette abondance est trompeuse et le repérage de tels manuscrits est, comme on va le voir, toujours très laborieux. Mais l'existence déjà connue de cinq manuscrits philosophiques clandestins ayant appartenu à Saint-Victor, dont deux sont conservés à la Bibliothèque Mazarine, les trois autres à la Bibliothèque Nationale permettait, si l'on en trouvait d'autres, de remettre en question la vision d'une bibliothèque toute entière tournée vers le passé et fermée aux audaces et aux curiosités des Lumières.

L'allusion récurrente, quand il est question de la bibliothèque de Saint-Victor au XVIII ${ }^{\mathrm{e}}$ siècle, à celles des Lettres persanes qui sont consacrées à la visite du persan Rica dans ce célèbre lieu parisien m'a engagée à relire les commentaires d'autres visiteurs contemporains de Montesquieu. Je commencerai par évoquer ces quelques témoignages, puis je ferai le bilan des identifications que j'ai pu faire de manuscrits philosophiques clandestins ayant appartenu à la bibliothèque, et enfin je présenterai rapidement les idées qu'ils introduisaient à Saint Victor.

\section{Les témoignages}

Revenons quelques instants sur celui, très célèbre, de Montesquieu, ou plutôt de son personnage romanesque, Rica, dans cinq des Lettres persanes. ${ }^{5}$ La tradition veut que la bibliothèque qui y est décrite sans être nommée soit celle de Saint-Victor, mais non sans quelques réserves. D'autres hypothèses ont été proposées. Henri Roddier ${ }^{6}$ songe à la bibliothèque de l'Oratoire, dont le

\footnotetext{
${ }^{2}$ Ira O. Wade, The Clandestine Organization and Diffusion of Philosophic Ideas in France from 1700 to 1750, Princeton University Press, 1938, rééd. New York, Octagon Books Inc., 1967.

3 Miguel Benítez, La Face cachée des Lumières. Recherches sur les manuscrits philosophiques clandestins de l'âge classique, Paris, Universitas, Oxford, The Voltaire Foundation, 1996.

${ }^{4}$ La Lettre clandestine, $\mathrm{n}^{\circ} 1$ à 16, Paris, PUPS, 1992-2008. Cette revue annuelle publie un dossier thématique sur la littérature philosophique clandestine à l'Âge classique et fournit une bibliographie exhaustive, des études, une documentation, des informations sur les recherches qui sont conduites dans le monde entier à son sujet (pups.paris-sorbonne.fr).

${ }_{5}$ Montesquieu, Lettres persanes, lettres 133-137, voir notamment les éditions de Paul Vernière (Classiques Garnier, 1960), Antoine Adam (Droz, TLF, 1965), Laurent Versini, (GF, 1995).

${ }^{6}$ Henri Roddier, «De la composition de L'Esprit des Lois. Montesquieu et les oratoriens de l'Académie de Juilly » Revue d'Histoire littéraire de la France, 1952, p. 439-450.
} 
bibliothécaire est l'abbé Desmolets ami de Montesquieu. Paul Vernière ${ }^{7}$ insiste sur l'erreur qui consisterait à interpréter cette visite comme une visite réelle, que ce soit à Saint-Victor ou à l'Oratoire, parce que toutes les bibliothèques sont rangées par format et que le classement par matières, inauguré par l'Oratoire, est généralisé dès la fin du XVII ${ }^{\mathrm{e}}$ siècle. Son hypothèse, séduisante, est qu'il s'agit d'un résumé des richesses de la Brède au moment où Montesquieu commence à en faire l'inventaire avec l'abbé Bottereau-Duval. Mais la ressemblance avec Saint-Victor n'est pas fortuite et elle vient en droite ligne du modèle le plus reconnu de Montesquieu, L'Espion du grand seigneur, titre d'un recueil de lettres et mémoires du Gênois Giovanni Paolo Marana (1642-1693), publié en 1684. Son héros, l'espion turc, visite à Paris «une célèbre bibliothèque qu'il y a en cette ville, sous la direction de certains religieux qui à certaines heures du jour sont obligés de servir tous ceux qui veulent s'asseoir et étudier $»^{8}$.

A côté de ces visiteurs fictifs, il est bon de citer un témoin réel. En 1698, Martin Lister (1638-1712), médecin et naturaliste anglais, accompagne le comte de Portland (Hans Willem Bentinck 1648-1709) dans son ambassade auprès de Louis XIV après la paix de Ryswick qui marque la fin de la coalition d'Augsbourg (16881697). De retour en Angleterre il publie le récit de son Voyage à Paris. Un classement méthodique réserve le cinquième des onze chapitres aux bibliothèques publiques. Une longue rubrique est réservée à celle qui nous intéresse aujourd'hui :

J'ai vu la bibliothèque de Saint-Victor. Cette très ancienne abbaye est la mieux située de tout Paris, avec de très grands jardins et des allées d'arbres, bien tenues. La bibliothèque est une grande et belle galerie : trois fois par semaine elle est ouverte au public, qui y trouve sur une longue table à double pupitre, tout ce qu'il faut pour écrire commodément pour quarante ou cinquante personnes. Le catalogue n'en était pas terminé, et on ne comptait pas l'imprimer: c'est cependant ce que l'on devrait faire dans tous ces établissements pour empêcher les livres de se perdre, pour la plus grande commodité des étrangers et pour y faire mention des bienfaiteurs.

Au bout de cette galerie, sont serrés les manuscrits : on dit qu'il y en a trois mille, et, sans être fort anciens, ils ont bien servi à donner des éditions correctes de plusieurs auteurs. C'est l'un des lieux les plus agréables que l'on puisse voir par la beauté de la vue, le calme et l'absence du bruit au milieu d'une si grande ville. ${ }^{9}$

Outre l'agrément des lieux on retiendra le souci de la commodité du lecteur et de l'efficacité de son travail ainsi que l'intérêt et l'utilité des manuscrits pour «les gens de savoir ». L'allusion aux éditions établies à partir des manuscrits de SaintVictor suggère une visite guidée par un bibliothécaire soucieux du rayonnement de sa bibliothèque. On songe alors à la petite note collée dans un catalogue manuscrit

\footnotetext{
${ }^{7}$ Voir ci-dessus, note 5.

${ }^{8}$ Giovanni Paolo Marana, L'Espion turc dans les cours des Princes chrétiens ou Lettres et mémoires..., Cologne, 1696, t. II, p. 356.

${ }^{9}$ Voyage de Lister à Paris en MDCXCVIII traduit pour la première fois, publié et annoté par la société des bibliophiles français, Paris, 1873.
} 
(Mazarine 4202) ${ }^{10}$, dont je parlerai plus tard, où l'on peut lire les instructions suivantes : «les plus anciens manuscrits ... Manuscrits à faire voir aux étrangers ${ }^{11}$.

L'intérêt et l'utilité des manuscrits, c'est bien ce que met en évidence un autre témoignage réel. Robert Challe (1659-1721), romancier, mémorialiste et philosophe déiste est l'auteur d'un des plus célèbres manuscrits philosophiques clandestins, intitulé Difficultés sur la religion proposées au père Malebranche, rédigé vers 1710, resté inédit jusqu'en 1768, date à laquelle un abrégé défiguré en est publié par D'Holbach sous le titre de Militaire Philosophe. On apprend par sa correspondance avec les auteurs du Journal littéraire ${ }^{12}$ de La Haye qu'il a été un lecteur assidu de la bibliothèque de Saint Victor. Encouragé par un compte rendu élogieux qu'ils ont fait de son chef-d'œuvre romanesque publié en 1713, Les Illustres Françaises, Challe entretient les journalistes de «tablettes chronologiques » qu'il cherche à publier. Le 22 janvier 1714 il leur parle de cet ouvrage qui lui «a coûté près de deux ans de travail » et «une infinité de visites dans les plus fameuses bibliothèques de Paris, pour puiser dans les originaux anciens tant imprimés que manuscrits ». Le 22 juillet 1718, il précise : «Ces Tablettes sont mon ouvrage favori, et qui m'ont coûté une très forte application sur plusieurs manuscrits de $\mathrm{S}$. Victor et de Sainte Geneviève ${ }^{13}$.

La bibliothèque de Saint-Victor est ici mentionnée comme une des sources de la documentation d'un travail historique érudit et critique qui présente un « raccourci de ce qui s'est passé depuis la naissance de Jésus-Christ jusques à l'année 1702 »ò Challe dit qu'il suit et corrige le plan des Tablettes chronologiques de Guillaume Marcel $(1682)^{14}$. Les Tablettes chronologiques de Challe n'ont pas été retrouvées mais, d'après ce que l'auteur en dit, ses principales cibles sont l'Église, la papauté, les jésuites, le monachisme, les Conciles, notamment celui de Constance (14141418). Il est fort probable que Challe étudie à Saint-Victor les manuscrits concernant les conciles dont il dit dans les Difficultés : «On ne sait jamais si on a des copies fidèles et entières. On y ajoute, on en retranche, on a trois ou quatre copies

\footnotetext{
${ }^{10}$ Mazarine 4202, papier collé à la fin : « les plus anciens manuscrits : $n^{\circ} 1024$ et 214 . Voyez la dissertation $n^{\circ} 889$, citation fausse. Voyez notes sur le $n^{\circ} 1024$. Manuscrits à faire voir aux étrangers ».

${ }^{11}$ En 1773, un grand seigneur italien, et savant, Alfonso Bonfioli Malvezzi, effectue un voyage en Europe. D'après son journal, les bibliothèques parisiennes font partie du tour obligé et celle de Saint-Victor y est mentionnée : «Les bibliothèques les plus curieuses sont : 1 la bibliothèque du Roi ; 2 celle de Saint Germain des Prés ; 3 celle du collège Mazarin ; 4 celle de Sainte Geneviève, 5 celle de Saint Victor », Viaggio in Europa e altri scritti, Ferrare, 1988.

${ }^{12}$ Prosper Marchand, s'Gravesande, Van Effen, Sallengre, Alexandre, Saint-Hyacinthe.

${ }^{13}$ Robert Challe, Mémoires, correspondance complète, éd. de F. Deloffre et J. Popin, Genève, Droz, 1996, p. 476 et 515.

${ }^{14}$ Guillaume Marcel, avocat au Parlement de Paris, Tablettes chronologiques contenant par ordre l'état de l'Église en Orient et en Occident, les auteurs ecclésiastiques, les schismes, les hérésies et opinions qui ont été condamnées, Paris, P. Thierry, 1682.
} 
différentes de celui de Constance. Au fond les conciles supposent la religion et ne la prouvent point $»^{15}$.

Du point de vue de l'usage de la bibliothèque de Saint-Victor que pouvait faire le public cultivé, il est intéressant d'apprendre que, dans la première décennie du XVIII ${ }^{\mathrm{e}}$ siècle, Challe y a cherché et trouvé des matériaux pour un ouvrage critique sur l'Église de Rome dont l'inspiration inaugure un aspect essentiel de la pensée des Lumières. Les chanoines se doutaient-ils que ce lecteur assidu de manuscrits sur l'histoire de l'Église préparait des Tablettes chronologiques pour lesquelles il chercherait un éditeur dans le Refuge ? On ne le saura jamais. En tout cas ses confidences aux journalistes de La Haye qui ne le connaissent que sous la seule signature de «l'auteur des Illustres Françaises» sont bien peu compromettantes. Cependant il est évident pour le lecteur d'aujourd'hui que ses lectures à Saint-Victor n'ont pas servi de sources qu'aux Tablettes chronologiques et que Challe y a puisé nombre d'arguments contre les dogmes, les sacrements, les ordres religieux, la morale chrétienne, l'Écriture. Ce que nous ne savons pas c'est s'il a lu à Saint-Victor des manuscrits philosophiques clandestins. Ce que nous pouvons affirmer c'est qu'il y en avait, et plus que les cinq que j'ai déjà évoqués. Voici donc le bilan des identifications de manuscrits philosophiques clandestins que je me réjouis d'avoir pu faire à l'occasion de cette recherche.

\section{Enquête et identifications}

J'en ai en effet trouvé cinq de plus, sans compter trois manuscrits aux titres incomplets mais ressemblants et dont le contexte permet de faire l'hypothèse qu'ils appartiennent bien au même corpus. Ce qui fait en tout dix manuscrits philosophiques clandestins (peut-être treize), c'est une quantité significative. Sans entrer dans le détail de la quête difficile qu'il a fallu pour arriver à ce résultat, je me contenterai de dire que, même dans une bibliothèque bien réelle avec un catalogue imprimé des manuscrits, la recherche d'un manuscrit philosophique clandestin s'apparente à celle d'une aiguille dans une botte de foin. Ces manuscrits sont pour la plupart anonymes, ils ont des titres très variables d'une copie à l'autre, ils sont rassemblés dans des recueils, ou, ce qui est pire, mélangés à des textes de natures très diverses, il en existe des abrégés ou des extraits qui ne sont pas toujours faciles à distinguer. Le titre du recueil, quand il y en a un, est souvent celui de la première pièce du recueil. L'identification n'est bien souvent certaine qu'en vérifiant le texte, ce qui dans le cas d'une bibliothèque disparue n'est guère possible. En dépit de toutes ces difficultés, on peut se réjouir que la bibliothèque Mazarine conserve sous la cote 4202 le catalogue manuscrit des manuscrits dont on se servait à Saint-Victor depuis le commencement du XVIII ${ }^{\mathrm{e}}$ siècle, d'après Alfred Franklin ${ }^{16}$.

Je n'ai pas trouvé dans ce catalogue deux des quatre manuscrits philosophiques clandestins que je connaissais, Le ciel ouvert à tous les hommes (BnF fr. 25290) et La Béatitude des chrétiens ou le fléau de la foi (Mazarine 1072) et à ce stade de mon enquête je ne peux pas expliquer pourquoi. Mais parmi les cinq

15 Robert Challe, Difficultés sur la religion proposées au Père Malebranche, éd. de F. Deloffre et F. Moureau, Genève, Droz, 2000, p. 489-490.

${ }_{16}$ Alfred Franklin (1830-1917), Histoire de la bibliothèque de l'abbaye de Saint Victor à Paris, d'après des documents inédités, Paris, A. Aubry, 1865. 
manuscrits dont j'ai découvert la trace, il y a un deuxième exemplaire du Ciel ouvert à tous les hommes. On peut l'affirmer sans risque d'erreur puisque la copie de la Bibliothèque nationale est isolée sous sa reliure, alors que la copie signalée sur le catalogue 4202 est la première des neuf pièces d'un recueil. Les huit autres manuscrits ne figurent qu'en un seul exemplaire. Le bilan est particulièrement appréciable car les neuf textes identifiés (en dix copies) constituent un échantillon très représentatif du corpus clandestin, à commencer par la façon dont ils se présentent au lecteur : cinq d'entre eux, donc la moitié, sont dans des recueils, ce qui constitue une proportion significative.

Je les ai tous trouvés dans les ouvrages en prose in $4^{\circ}$ entre le numéro 1368 et le numéro 1419, puis, en raison d'une lacune, entre les numéros 1477 et 1779. Ils représentent à eux neuf (je parle ici des titres et non des copies) les deux types caractéristiques du corpus. Ce qu'on pourrait appeler le noyau dur, qui correspond littéralement à la découverte de Lanson en 1912, est constitué de cinq productions clandestines de la toute fin du XVII ${ }^{\mathrm{e}}$ siècle et du début du XVIII ${ }^{\mathrm{e}}$ siècle :

1369 Essai de métaphysique dans les principes de B. de SP $1^{\text {ère }}$ partie

1368 Traité des trois imposteurs

De tribus impostoribus anno MDIIC

1371 Le Ciel ouvert à tous les hommes

1384 Examen de la religion dont on cherche l'éclaircissement de bonne foy

Quatre d'entre eux, rédigés en français entre 1690 et 1730, sont parmi les plus célèbres et les plus répandus. Ils ont connu une large diffusion, ont très vite été recherchés et ont exercé une grande influence. Un seul d'entre eux, l'Essai de métaphysique, a été édité précocement, en 1731, l'Examen l'a été en 1745, Le Ciel ouvert en 1768, ce qui est aussi l'année de l'édition de référence du Traité des trois imposteurs dont l'histoire éditoriale, commencée en 1719, est très particulière et n'a pas sa place ici. Seul Le Ciel ouvert est clairement signé. L'auteur, Pierre Cuppé, est curé de Bois, diocèse de Saintes, chanoine régulier de Saint-Augustin. On ne s'étonne pas qu'il y en ait deux exemplaires à Saint-Victor. L'Essai de métaphysique est attribué à Boulainvilliers par les initiales MLCDB. Les deux autres manuscrits sont anonymes. Il faut rattacher à ce bloc de quatre manuscrits français le De tribus impostoribus anno MDIIC relié à la suite du Traité des trois imposteurs dans le même recueil 1368. Il est faussement daté de 1598. C'est un traité déiste rédigé en latin au XVII ${ }^{\mathrm{e}}$ siècle qui a surtout beaucoup circulé en Allemagne et dans les pays du Nord. Sur quatre-vingt-quinze copies actuellement connues, il n'y en a que deux en France, celle de Saint-Victor, actuellement conservée à la $\mathrm{BnF}$ (fr. 25290) et une à Strasbourg (BNU). Il a été imprimé à Vienne en 1753. Les chanoines possédaient donc une rareté. L'avoir rapproché du Traité des trois imposteurs prouve une bonne connaissance de la légende qui courait sur un De tribus impostoribus qui aurait été écrit (ou commandé) en 1230 par l'empereur d'Occident Frédéric II (1194-1250). La copie des Trois imposteurs du recueil 1368 contient d'ailleurs toutes les références des ouvrages qui résument l'affaire.

Les quatre autres manuscrits sont des copies du XVIII ${ }^{\mathrm{e}}$ siècle de textes antérieurs à la période commune de rédaction du corpus clandestin découvert et 
défini par Lanson. Deux sont assurément du $\mathrm{XVI}^{\mathrm{e}}$ siècle. Le Tractatus de immortalitate animae de Pomponazzi ( $\left.{ }^{\circ} 1770\right)$, publié en 1516, a attiré l'attention de l'Inquisition sur son auteur, philosophe aristotélicien. Ce traité est une des sources explicites des manuscrits philosophiques clandestins en particulier par l'intermédiaire du Dictionnaire historique et critique de Bayle (1697). On en connaît deux autres copies (Copenhague et Wolfenbüttel). À ma connaissance l'exemplaire de Saint-Victor est le seul repéré en France pour l'instant. Le sujet de l'immortalité de l'âme, on va le voir, est un des thèmes récurrents de la littérature clandestine. La Béatitude des Chrétiens ou Le fléau de la Foy (Mazarine 1072), dont l'auteur Geoffroy Vallée (1535-1574 ) a été condamné à mort et exécuté en 1574 pour l'avoir écrit, a été publié en 1573. Ce libelle athée original est une rareté bibliographique, on ne connaît qu'un exemplaire de l'édition de 1573, retrouvé par Bernard de La Monnoye en 1710, aujourd'hui conservé à la bibliothèque Méjanes d'Aix-en-Provence. On en connaît une vingtaine de copies manuscrites, toutes, sauf une, postérieures à la découverte de l'imprimé. Le manuscrit de Saint-Victor est complet et bien documenté, il comporte une notice autographe de Camille Falconet sur l'histoire du manuscrit, et la copie de l'arrêt du Parlement. Quant au Colloque de Jean Bodin entre sept savants, traduction française du fameux Colloquium heptaplomeres, il n'a pas été publié avant 1914, et sous forme d'extraits seulement. Sa date est incertaine. La première édition latine date de 1857 . On en connaît cent vingt copies en latin mais le texte français est rare. On n'en connaît que six copies dont celle de Saint-Victor, copie du XVIII ${ }^{\mathrm{e}}$ siècle conservée à la Bibliothèque Mazarine. Si on s'en tient à l'attribution à Jean Bodin (1530-1596) il est du XVI siècle. Mais cette attribution est contestée par certains savants qui datent le texte du XVII ${ }^{\mathrm{e}}$ siècle (pas avant 1623). La discussion est vive.

Plus atypique est L'Autre Monde ou les états et empires de la lune $\left(n^{\circ} 1371\right)$. Son originalité même est une des caractéristiques du corpus clandestin, qui, on le voit, n'est pas uniforme. Il s'agit du roman utopique de Cyrano de Bergerac (1619-1655), publié sous une forme expurgée de sa philosophie libertine en 1657. On connaît la version intégrale par les manuscrits qui sont au nombre de trois auxquels s'ajoute maintenant celui de Saint-Victor. Sa présence dans un recueil ( $\mathrm{n}^{\circ}$ 1371) où l'on trouve Le Ciel ouvert à tous les hommes de Pierre Cuppé est significative. Elle dénote une attention particulière au contenu, quelle que soit sa forme, et un jugement sûr, peut-être aussi une tradition orale, parce que le rapprochement ne peut être fortuit. Cette constatation conduit à s'intéresser de près aux recueils qui contiennent, sous la même couverture que des manuscrits philosophiques clandestins, des textes aux titres approchants. J'en retiendrai trois, que je ne peux encore, dans l'état de mes recherches, ni rejeter ni annexer. Dans le recueil 1369, juste après l'Essai de métaphysique dans les principes de Benoît de Spinoza, la dernière pièce s'intitule: Remarques abrégées sur l'apocalypse, elle est décrite comme incomplète, il pourrait s'agir d'extraits des Remarques critiques sur le Nouveau Testament. Dans le recueil 1371 qui contient Le Ciel ouvert... de Cuppé et L'Autre Monde... de Cyrano, la deuxième pièce est annoncée par un titre tronqué : De la religion chrétienne... Il pourrait s'agir d'extraits de La Religion chrétienne analysée, manuscrit anonyme de large diffusion. Enfin dans le recueil 1372 qui 
succède à plusieurs recueils fournis en manuscrits clandestins, une série de titres de chapitres évoque des extraits de l'Abrégé d'histoire universelle de Boulainvilliers ${ }^{17}$. L'enquête doit donc se poursuivre de ce côté-là. Les réponses qu'elle donnera changeront éventuellement le bilan quantitatif, mais ne changeront rien aux idées que les dix manuscrits introduisaient à Saint-Victor.

\section{Les idées introduites}

D'abord il faut préciser qu'une bonne quantité des manuscrits philosophiques clandestins qui sont conservés dans nos bibliothèques publiques viennent d'institutions religieuses: de séminaires, de couvents, d'abbayes, de collèges, du chapitre de Notre-Dame de Paris, de la Sorbonne, etc. La possession de semblables manuscrits ne conduit pas nécessairement les membres du clergé à devenir hétérodoxes comme Pierre Cuppé, chanoine régulier de Saint-Augustin, ni matérialiste athée comme Jean Meslier, curé d'Étrépigny en Champagne, mort en 1729 après avoir rédigé et copié en trois exemplaires ses Mémoires et Sentiments sur une partie des erreurs et des abus de la conduite et du gouvernement des hommes conservés à la Bibliothèque Nationale.

Ces lectures sont évidemment nécessaires pour préparer le discours apologétique, la réplique immédiate et même tout simplement les sermons. C'est peut-être une des raisons pour lesquelles on trouve des manuscrits philosophiques clandestins dans des recueils intitulés recueils de sermons. Ce n'est le cas, à SaintVictor, que de l'Essai de métaphysique dans les principes de Benoit de Spinoza, neuvième pièce d'un "recueil de sermons et instructions sur différents sujets " (1369), dans lequel un «exercice de retraite» voisine avec des documents sur le procès entre le Père Girard et La Cadière ${ }^{18}$ ainsi qu'avec des chansons et des vers sur la Constitution Unigenitus. Quatre autres des manuscrits que j'ai identifiés se trouvent dans des recueils sans titres mais après une série de sept recueils de sermons (1261 à 1267). Les cinq autres manuscrits, on l'a vu, sont isolés sous leur reliure. Rien ne les dissimule ni les uns ni les autres à la curiosité du lecteur. Il reste que deux manuscrits conservés avec l'estampille de Saint-Victor ne figurent pas sur le catalogue 4202. On ne peut cependant pas voir dans cette absence une dissimulation volontaire par prudence. Pourquoi en effet cacher Le Ciel ouvert à tous les hommes, puisqu'un autre exemplaire du même traité est clairement désigné par son titre dans le recueil 1371 ? Pourquoi aussi cacher La Béatitude des Chrétiens qui n'est pas un manuscrit plus dangereux que les neuf autres? L'explication est ailleurs, peut être dans les paquets et cartons que signale une liasse glissée dans le catalogue 4202.

Je n'ai pour l'instant tiré qu'un autre renseignement de la consultation de ce catalogue 4202: à côté du titre du Colloque de Jean Bodin entre sept savants on

\footnotetext{
${ }^{17}$ Dans le même recueil signalé au numéro 1372 on trouve des « remarques sur quelques livres de Tertullien », or ce Père de l'Église est une des sources les plus fréquentes de l'érudition clandestine.

${ }^{18}$ Un procès scandaleux eut lieu en 1731 à Aix-en-Provence entre le père Girard, jésuite, et une de ses pénitentes, Marie-Catherine Cadière.
} 
peut lire l'appréciation «Dangereux ». Cette mention est d'autant plus remarquable qu'elle paraît isolée. Je n'ai en effet trouvé de commentaire d'aucune sorte sur aucun autre de nos manuscrits. Cette précision exceptionnelle pourrait s'interpréter comme une mise en garde contre le préjugé favorable que le nom d'un auteur réputé risque d'éveiller à l'égard d'un ouvrage dont les apparences sont plus savantes et moins visiblement subversives que celles des autres manuscrits inventoriés. Ce danger que signale le bibliothécaire à propos du Colloque de Jean Bodin est bien la caractéristique commune aux dix manuscrits dont nous savons qu'ils ont appartenu à Saint-Victor. On ne peut parler pour aucun d'eux d'un système philosophique original, novateur. Ce qui résume le mieux les idées véhiculées par l'ensemble de ces textes, c'est une philosophie critique, violemment antireligieuse. Cette philosophie puise à toutes les sources, de l'Antiquité au Dictionnaire historique et critique de Bayle qui est littéralement pillé. Tous ces textes sont faits de collages d'œuvres diverses et d'emprunts réciproques. Tout se passe comme si chacun apportait consciencieusement sa contribution à une grande œuvre commune de doute généralisé. Une formidable contagion d'esprit critique contribue à l'élaboration à la fois patiente et passionnée d'un message universel. L'anonymat, la circulation manuscrite clandestine ne sont pas seulement dus à la prudence, à la crainte de la censure mais d'abord à l'absence d'ambition littéraire. Plus tard, dans les années soixante, Voltaire et les Encyclopédistes s'occuperont de la mise en forme. Ils couperont, rogneront, résumeront, corrigeront, orneront et publieront tout ce qu'ils trouveront. En attendant, les tâcherons des Lumières préparent dans l'obscurité les arguments, fourbissent les armes avec des examens, des analyses, des essais sur Dieu, sur l'âme, sur la formation du monde, sur l'imposture sacerdotale.

Les chanoines de Saint-Victor sont bien renseignés. Leur échantillonnage est suffisant pour connaître, voire combattre, les idées qui circulent : rationalisme du Ciel ouvert, spinozisme de l'Essai de métaphysique, déisme du Colloque de Bodin et du De tribus impostoribus, athéisme de la Béatitude des Chrétiens, matérialisme inspiré de spinozisme du Traité des trois imposteurs. Quant à l'Examen de la religion, en l'absence du texte que le titre désigne, on ne peut rien en dire si ce n'est qu'il fait partie d'un ensemble de plus de soixante copies réparties en deux familles principales, elles-mêmes scindées en plusieurs versions et ainsi de suite. Il suffit pour comprendre la fructueuse complexité de la transmission manuscrite de lire ce que dit à son sujet un copiste plein d'humour :

J'ai vu cet écrit pencher tantôt vers le déisme, tantôt vers l'athéisme, tantôt vers le scepticisme... Mais de tous ces changements je crois pouvoir assurer qu'il n'en est point de plus considérables que ceux que j'ai cru devoir y faire : je ne sais même s'ils ne m'autorisent pas à le présenter comme un ouvrage totalement neuf et original. ${ }^{19}$

${ }^{19}$ Questions d'un homme raisonnable et de bonne foi sur la religion chrétienne dont il cherche à s'instruire, par J.K.L.M., $3^{e}$ fils d'Alfa, manuscrit en 12 chapitres, Bibliothèque de Gand, ms. 284. 
Ce plaisant commentaire ne caractérise pas seulement l'Examen de la religion, il dépeint fort bien la malléabilité des manuscrits et la constante versatilité de la pensée clandestine. C'est contre ce danger protéiforme que les chanoines de Saint-Victor se prémunissent avec le choix judicieux qu'ils proposent à leur lecteur. Toutes les tendances sont abondamment représentées dans leur bibliothèque. Le recueil manuscrit 1368 conservé à la Bibliothèque nationale (fr. 25290) et le catalogue imprimé des manuscrits de la Bibliothèque nationale fournissent deux informations qui ne figurent pas sur le catalogue des manuscrits de Saint-Victor conservé à la Mazarine (4202). On lit sur le catalogue de la Bibliothèque nationale «Cours de philosophie à Saint-Victor en $1762 »$, ce qui ne figure pas sur le manuscrit dont la première page indique seulement «formé en recueil 1762 ». Le contenu ne convient pas exactement à un cours. Je pense, d'après le détail du recueil, que le Traité des trois imposteurs et le De tribus impostoribus ont été réunis à la physique, la métaphysique, la logique et l'éthique qui les précèdent et forment à elles seules un cours de philosophie. Le recueil ainsi constitué en 1762 n'est pas un cours à proprement parler, mais son but pédagogique n'a pas échappé au rédacteur du catalogue de la Nationale. Le Traité des trois imposteurs, un des plus anciens, des plus répandus et des plus célèbres manuscrits philosophiques clandestins est une lecture instructive sur la naissance du matérialisme du XVIII ${ }^{\mathrm{e}}$ siècle, sur la critique philosophique de l'idée de Dieu et sur l'influence de Spinoza ${ }^{20}$. Car ce n'est sans doute pas un hasard si on a relié, en 1762, le Traité des trois imposteurs à la suite de l'éthique, dernière partie du cours de philosophie. Le Traité clandestin s'inspire en effet de l'Éthique de Spinoza, en la transformant comme on le fait en France au début du XVIII ${ }^{\mathrm{e}}$ siècle en ce que Paul Vernière appelle «un spinozisme de contrebande». La constitution du recueil en 1762 intervient six ans avant l'édition des Trois Imposteurs suscitée par D'Holbach et Naigeon. Le manuscrit rédigé vers 1700 circulait avec succès depuis une soixantaine d'années, il devenait d'une certaine façon une lecture non seulement utile mais nécessaire.

C'est sur cet aspect que je voudrais conclure, conclure provisoirement seulement, car l'enquête ne fait que commencer. Il reste beaucoup de questions à poser, cependant une réponse est sûre: les chanoines de Saint-Victor étaient très bien renseignés sur la circulation clandestine des manuscrits philosophiques. Leurs acquisitions ne peuvent en aucun cas être le fruit du hasard. Il s'agit d'une collection de premier ordre où les plus grands titres sont représentés. La présence de copies de textes moins répandus, mais éclairants sur les sources, évoque une bonne connaissance des tendances critiques. La réunion de L'Autre Monde ou les états et empires de la lune de Cyrano avec Le Ciel ouvert à tous les hommes de Cuppé suggère une étude en profondeur du mouvement critique contemporain et de son étroite relation avec le libertinage érudit du siècle précédent. En faisant passer de cinq à dix le nombre de manuscrits du corpus clandestin possédés à Saint-Victor, cette enquête permet de répondre que les chanoines n'ont pas renoncé à tout dynamisme intellectuel et ne vivent pas repliés «sur un savoir suranné». On peut

${ }^{20}$ Paul Vernière, Spinoza et la pensée française avant la Révolution, Paris, PUF, 1954. 
bien parler de la Bibliothèque de Saint-Victor comme d'un témoin actif des Lumières.

Geneviève Artigas-Menant Université Paris 12-Val de Marne 